\title{
An Estimate of Relativistic H- Stripping from a Hot Diamond Foil Tomlin 3/21/05
}

An estimate is made for a $12 \mathrm{~mm} \times 14 \mathrm{~mm}$ diamond foil heated by the $\mathrm{H}$ - beam that it is stripping. Previous thermal calculations estimate operating temperatures of $>3000 \mathrm{~K}$. The method is to use the S.B. equation to get watts. Power is then converted to numbers of photons, then the photons are blue-shifted to two bands on either side of the peak BB wavelength that will strip one or both electrons. The bands very roughly have edges at $\sim 0.75 \mathrm{eV}$ and $\sim 13.6 \mathrm{eV}$. All the photons in the band are capable of stripping the electron. Then use the probability of stripping formula for comparison. The calculation dutifully remembers that one $\mathrm{BB}$ photon is still just one photon for statistical calculations after it is Doppler-shifted. The photon energy multiplier or Beta-multiplier simply boosts the photon to high enough energy to do some work.

Using the S.B. equation:

$$
\begin{aligned}
\mathrm{P} / \mathrm{A} & =\sigma \mathrm{T}^{4} \mathrm{~J} / \mathrm{m}^{2} \mathrm{~s} \quad \text { Stelan-Boltzmann Law } \\
\text { where } \sigma & =5.6703 \times 10^{-6} \text { watt } / \mathrm{m}^{2} \mathrm{~K}^{4}
\end{aligned}
$$

Diamond and graphite have emissivities ranging from 0.02 to 0.9 . The worst case, e = 1.0 , is used. There is 780 watts/second radiating from such a hot object of that size, given that the emissivity is 1 .

Wien's Displacement Law:

$$
\lambda_{\text {peak }} T=2898 \times 10^{-3} \mathrm{~m} \cdot \mathrm{K}
$$

Yields the peak of the black body curve at $966 \mathrm{~nm}$.

But the black body radiation is Doppler-shifted w.r.t. the $8 \mathrm{GeV}$ ions;

$\lambda_{f}=\lambda_{i} \cdot \gamma(1-\beta \cos \theta)$

The BB curve is blue-shifted so that 540 watts in the BB 300 to $1850 \mathrm{~nm}$ range is > 13.6 $\mathrm{eV}$, stripping the second electron, and 210 watts in the BB 1800 to 3900nm range gets shifted to $<300 \mathrm{~nm}$. It takes more long-wave photons than short-wave photons to make a watt, so we choose a wavelength of $966 \mathrm{~nm}$, the blackbody peak for the bluer band, and a wavelength of $2500 \mathrm{~nm}$ for the redder band, to convert to photons.

540 watts at $966 \mathrm{~nm}$ can strip both electrons, and 210 watts at 2500nm can strip the first electron. This is a rough, but reasonable approximation. Next we convert watts to number of photons lumping into two groups at 966 and $2500 \mathrm{~nm}$. First find the energy of each photon: 
$E=\frac{h c}{\lambda}=\frac{6.626 \times 10^{-34} \mathrm{Js} \cdot 2.998 \times 10^{8} \mathrm{~m} / \mathrm{s}}{2.5 \times 10^{-6} \mathrm{~m}}$

$E=7.95 \times 10^{-20}$ Joule $/$ photon At $2500 \mathrm{~nm}$

$E=2.06 \times 10^{-19}$ Joule $/$ photon At $966 \mathrm{~nm}$

$540 \mathrm{Joule} / \mathrm{sec}=540 \mathrm{Watts}, 966 \mathrm{~nm}$

There are $\frac{540 \mathrm{~J} / \mathrm{s}}{2.06 \times 10^{-19} \mathrm{~J} / \text { photon }}$ or

$2.62 \times 10^{21}$ photon / second at 966nm But the time is much shorter;

Next, estimate that the beam only sees the foil for about forty feet as it approaches it.

Estimate the interaction time as 40 nanoseconds. Keep in mind that this is an overestimate because the foil is thin, and we are artificially directing all the photons into a narrow beam that intercepts the ion beam. At $966 \mathrm{~nm}$ we have $\sim 1.05 \times 10^{14}$ blackbody photons that can arrive blue-shifted to UV while the beam is in the vicinity and strip both electrons. . At $2500 \mathrm{~nm}$ (210Watts) there are $\sim 1.06 \times 10^{14}$ blackbody photons that can arrive blue-shifted to visible while the beam is in the vicinity and strips the first electron.

Next, estimate that the beam only sees the foil for about forty feet as it approaches it. Estimate the interaction time as 40 nanoseconds. Keep in mind that this is an overestimate because the foil is thin, but we are directing all the photons into a narrow beam that intercepts the ion beam.

The intensity required to strip the first and second electron depends on the stripping cross section at a given wavelength. At $2500 \mathrm{~nm}$, the first electron has a cross section of about $2.8 \times 10^{-17} \mathrm{~cm}^{2}$. At $966 \mathrm{~nm}$ the first electron has a cross section of about $2.6 \times 10^{-17} \mathrm{~cm}^{2}$. The $13.6 \mathrm{eV}$ electron has a cross section in the UV of $1.5 \times 10^{-19} \mathrm{~cm}^{2}$.

Next we estimate how many photons it will take to strip some percentage of ions. We use the same 40 nsec IR time for this estimate. First the $13.6 \mathrm{eV}$ electron:

$$
I=\frac{\ln (0.999999999)}{-\sigma t}=\frac{-.000000001}{-1.5 \times 10^{-19} \mathrm{~cm}^{2} \cdot 40 \times 10^{-9} \mathrm{sec}}
$$

$$
I=1.67 \times 10^{17} \frac{\text { photons }}{\mathrm{cm}^{2} \text { sec ond }}
$$

So:

The net result here is that to strip $1 \mathrm{x} 10 \mathrm{e}-9$ of the $13.6 \mathrm{eV}$ electrons, we would need $1000 \mathrm{x}$ more photons than we actually see in those 40 nanoseconds. Or, we can say that $0.0000000001 \%$ of the $13.6 \mathrm{eV}$ electrons will be dissociated. 
The first electron is stripped by both the UV and visible photons, roughly $2.1 \times 10^{14}$ photons. Using the larger cross section for the $0.75 \mathrm{eV}$ first electron (another conservative estimate)

$$
I=\frac{\ln (0.999999999)}{-\sigma t}=\frac{-.000000001}{-2.8 \times 10^{-17} \mathrm{~cm}^{2} \cdot 40 \times 10^{-9} \mathrm{sec}}
$$

$I=8.9 \times 10^{14} \frac{\text { photons }}{\mathrm{cm}^{2} \text { second }}$

A somewhat larger percentage of the

$0.75 \mathrm{eV}$ electrons are stripped. 0.0000004\% are stripped.

This is an overestimate because of the several conservative assumptions applied here. A worst-case emissivity of one is used here. All of the photons are some-how directed to the interaction region, when in fact; the ion beam occupies a small fraction of a steradian of the BB radiation. The interaction region is 40 nanoseconds, long by any estimate. Finally, the estimated photo dissociation cross sections are generous.

For this rough calculation, $0.0000005 \%$ or $<5 \times 10 \mathrm{e}-8$ of the beam would be lost due to photoionization from blue-shifted black body radiation emitted by a 0.000168 square meter foil. Because of the generous estimates, it is likely that $<10 \mathrm{e}-11$ of the beam would actually be lost due to shifted BB radiation. 